\title{
Oclusão venosa central de retina na remissão de doença de Crohn: relato de caso
}

\author{
Retinal central vein occlusion in remission of Crohn's disease: case report
}

Iris de Souza Yamane ${ }^{1}$

Rafael da Silva Reis ${ }^{2}$

Haroldo Vieira de Moraes Jr. ${ }^{3}$
RESUMO

As doenças inflamatórias intestinais consistem na doença de Crohn e na retocolite ulcerativa. São caracterizadas pela inflamação crônica das alças intestinais. Os estudos e pesquisas sobre o processo inflamatório trouxeram avanços quanto ao tratamento destas doenças. As complicações sistêmicas das doenças inflamatórias intestinais envolvem muitos órgãos, incluindo os olhos. A verdadeira incidência de complicações oculares nas doenças inflamatórias intestinais é desconhecida, mas de acordo com a literatura varia de $3,5 \%$ a $11,8 \%$. As complicações oftalmológicas normalmente têm origem inflamatória. Os autores descrevem um caso clínico de uma paciente portadora de doença de Crohn que desenvolveu oclusão venosa central da retina (OVCR) e papiloflebite, sem nenhuma outra doença retiniana vascular associada, durante a fase de remissão da doença. O objetivo deste relato de caso é reforçar a importância da realização do exame oftalmológico completo de rotina nas doenças inflamatórias intestinais.

Descritores: Enteropatias inflamatórias; Colite ulcerativa; Doença de Crohn; Uveíte; Oclusão da veia retiniana; Feminino; Adulto; Relatos de casos [Tipo de publicação]

\section{INTRODUÇÃO}

As doenças inflamatórias intestinais (DII) são doenças crônicas e idiopáticas do trato gastro-intestinal, que se subdividem em doença de Crohn e em retocolite ulcerativa ${ }^{(1)}$. Fatores genéticos, infecciosos e imunológicos provavelmente influem na gênese da doença ${ }^{(1)}$. Atualmente, a diferenciação entre a doença de Crohn e a retocolite ulcerativa é baseada em critérios clínicos, endoscópicos e histopatológico. É importante ressaltar que em até $20 \%$ dos casos não é possível diferenciar as doenças inflamatórias intestinais entre si, neste caso são classificadas como "colites indeterminadas"(1).

Muitas manifestações extra-intestinais são conhecidas, dentre elas estão as alterações articulares, cutâneo-mucosas, hepático-biliares, renais, ósseas e oculares. A verdadeira incidência de complicações oculares nas doenças inflamatórias intestinais é desconhecida, mas de acordo com a literatura varia de $3,5 \%$ a $11,8 \%{ }^{(2-8)}$. Normalmente, uma grande variedade de patologias oculares de natureza inflamatória pode estar associada, acometendo os segmentos anterior e posterior do olho. Dentre as manifestações oculares no segmento anterior, se encontram conjuntivite, uveíte anterior, episclerite, infiltrados corneanos, entre outras. Já no segmento posterior, pode-se detectar pan-uveíte, neurorretinite, retinopatia serosa, oclusões vasculares, etc. Entretanto, a incidência de complicações retinianas em DII é rara chegando a menos de $1 \%{ }^{(2)}$. 
Descreve-se o caso clínico de uma paciente portadora de doença de Crohn que desenvolveu oclusão venosa central da retina (OVCR) e papiloflebite, sem nenhuma outra doença retiniana vascular associada, durante a fase de remissão da doença. O objetivo deste relato de caso é reforçar a importância da realização do exame oftalmológico completo de rotina nas DII.

\section{RELATO DE CASO}

MVS, sexo feminino, 32 anos, brasileira, com quatro anos de evolução da doença de Crohn, apresentou queda súbita da acuidade visual no olho direito em agosto de 2003. Nesta ocasião estava sem atividade da DII, pois apresentava exames laboratoriais sem alterações (VHS $=2$, proteína total $=6,7$, albumina $=57,1$, plaquetas $=266.000$, proteína $\mathrm{C}$ reativa $=0,96) . \mathrm{O}$ tratamento indicado para a paciente, na ocasião, era: Meticorten $^{\circledR} 15 \mathrm{mg} / \mathrm{dia}$ e Azatioprina $100 \mathrm{mg} / \mathrm{dia}$.

Em maio de 2003, a acuidade visual com correção era de $20 / 400$ no olho direito e 20/20 no olho esquerdo. Os exames de biomicroscopia e tonometria não apresentavam alterações. À fundoscopia do olho direito foi detectado edema de disco, congestão vascular, presença de exsudatos lipídicos e hemorragias peripapilares e em todo o pólo posterior. Já no olho esquerdo, o exame de fundo de olho não apresentava alterações. Os exames de retinografia (Figura 1) e angiografia fluoresceínica (Figuras 2 e 3) mostraram sinais de oclusão da veia central da retina, tipo papiloflebite aguda, no olho direito. Devido ao quadro clínico ocular, a dose do corticóide foi aumentada para $25 \mathrm{mg} / \mathrm{dia}$ e a da Azatioprina para $150 \mathrm{mg} / \mathrm{dia}$, porém não houve melhora da sintomatologia ocular.

Em maio de 2005, a acuidade visual com correção era de vultos no olho direito e 20/20 no olho esquerdo. O exame do segmento anterior se mantinha normal em ambos os olhos, mas no segmento posterior do olho direito havia vasos retinianos venosos dilatados, com aumento da tortuosidade e presença de hemorragias não isquêmicas. Portanto, o diagnóstico de OVCR e papiloflebite foi confirmado através dos exames de retinografia e angiografia fluoresceínica.

\section{DISCUSSÃO}

A OVCR em adultos jovens que cursam com queda aguda da acuidade visual ou fotopsia tem sido chamada de papiloflebite, caracterizada por edema de disco unilateral, grande aumento da tortuosidade vascular, dilatação das maiores veias retinianas e hemorragia retiniana ${ }^{(9)}$, ou seja, uma OVCR não isquêmica.

Já nos pacientes idosos, a OVCR geralmente está associada a anormalidades sistêmicas tais como: hipertensão arterial, diabetes e doenças vasculares sistêmicas ${ }^{(10)}$.

Ainda que a doença de Crohn e a retocolite ulcerativa sejam DII distintas, ambas podem cursar com trombose em qualquer leito vascular ${ }^{(11-12)}$. Na doença de Crohn, as alterações vasculares retinianas como oclusão de ramo ${ }^{(13)}$, flebi-

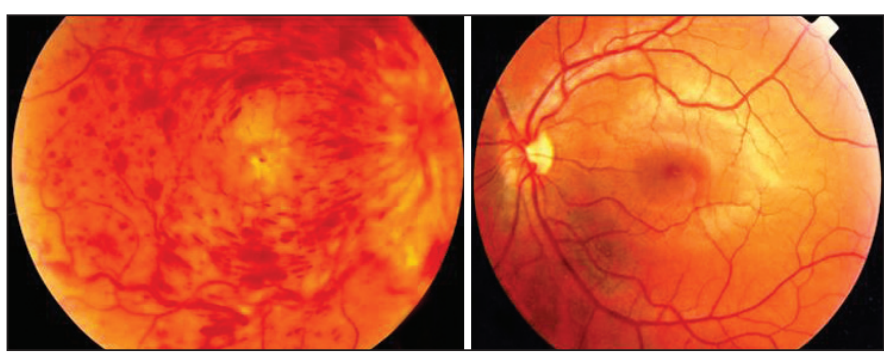

Figura 1 - Retinografia - Olho direito: sinais de OVCR com hemorragias retinianas difusas, alterações de calibre e trajeto vascular e importante edema de disco óptico. Olho esquerdo: sem alterações.

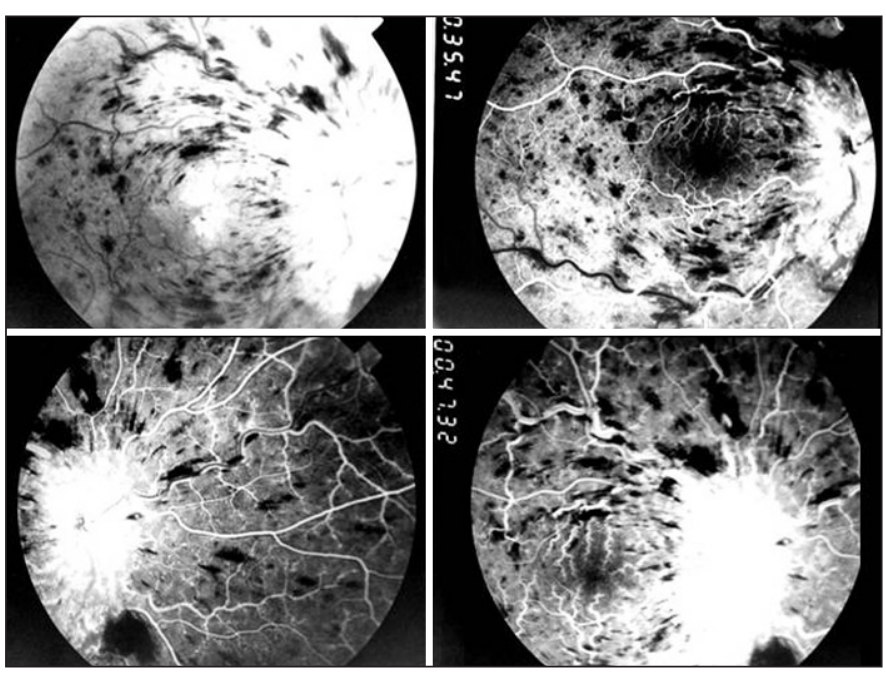

Figura 2 - Angiografia fluoresceínica (olho direito) - sinais de oclusão da veia central da retina, tipo papiloflebite aguda
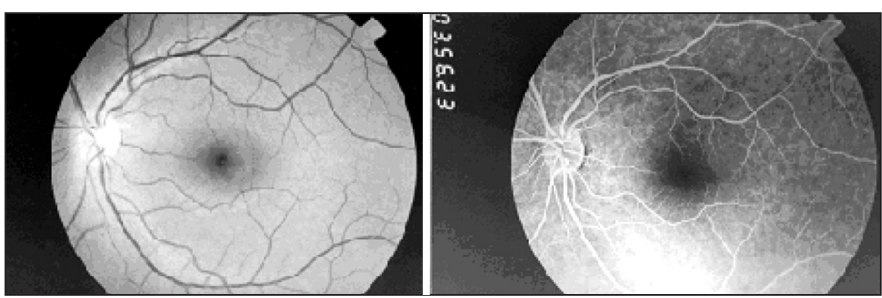

Figura 3 - Angiografia fluoresceínica (olho esquerdo) - sem alterações

te ${ }^{(14)}$ e $\mathrm{OVCR}^{(15)}$, têm sido relatadas em pacientes com DII em atividade, ou seja, com anormalidades de coagulação (aumento dos níveis de plaquetas, fator V e VIII, fibrinogênio e queda de antitrombina III) $)^{(12)}$.

No caso clínico descrito, a paciente apresentava-se em fase de remissão da DII comprovado por exames laboratoriais. Não foram detectados sintomas de qualquer outra doença vascular retiniana e tampouco evidências de outras causas de OVCR que não seja a doença de Crohn. Os exames laboratoriais e os exames clínicos realizados em conjunto pelos serviços de pelo gastroenterologia e oftalmologia confirmaram este diagnóstico.

A efetiva terapia para doenças vasculares retinianas na 
doença de Crohn não foi estabelecida ainda ${ }^{(11)}$. A corticoterapia sistêmica tem sido usada para tratar episódios de tromboembolismo na doença de $\mathrm{Crohn}^{(13-14)}$. Na OVCR pode haver remissão espontânea, mas como em $40 \%$ dos casos o prognóstico é ruím, a corticoterapia $\left(\right.$ Meticorten $\left.^{\circledR}\right)$ foi indicada. O tratamento com imunossupressor (Azatioprina) foi prescrito pela gastroenterologia.

A doença arterial retiniana não acompanhou a OVCR neste caso, mas a doença de Crohn também pode induzir alterações arteriais retinianas. O prognóstico das alterações arteriais também é reservado ${ }^{(11,16-17)}$.

No caso clínico descrito, a OVCR ocorreu no período de remissão da doença de Crohn. A paciente, portadora de doença de Crohn há 4 anos, não apresentava sintomas (como diarréia, anemia, febre...), nem sinais clínicos e/ou laboratoriais de atividade da doença.

Devido ao fato das DII apresentarem alta prevalência em jovens, com risco de possíveis complicações visuais e futuras seqüelas para o paciente, sugere-se o fortalecimento da relação multidisciplinar entre médicos gastroenterologistas, proctologistas e oftalmologistas.

Portanto, a realização do exame oftalmológico completo de rotina nas DII, tanto nas fases de atividade da doença como nas fases de remissão, torna-se essencial para o diagnóstico e tratamento precoce das alterações oculares.

\section{ABSTR ACT}

Inflammatory bowel disease (IBD) includes Crohn's disease and ulcerative colitis. Both diseases are characterized by chronic inflammation of the intestines. The advances in understanding the inflammatory process improved the treatment of inflammatory bowel disease. The systemic complications of inflammatory bowel disease involve several organs included the eyes. The incidence of eye complications in IBD is unknown, but according to the literature the incidence vary between $3.5 \%$ and $11.8 \%$. The ophthalmic complications are usually of inflammatory origin. We report a case of a patient with Crohn's disease that developed central retinal vein occlusion and papillophlebitis without any other retinal vascular disease during remission. The aim of this paper is to reinforce the importance of complete ophthalmic examination perform as a routine in patients with inflammatory bowel disease.

Keywords: Inflammatory bowel diseases; Colitis, ulcerative; Crohn disease; Uveitis; Retinal vein occlusion; Female; Adult; Case reports [Publication type]

\section{REFERÊNCIAS}

1. Tierney M Jr, McPhee SJ. Current medical diagnosis and treatment. New York: Lange Medical Books; 2002.

2. Ghanchi FD, Rembacken BJ. Inflammatory bowel disease and the eye. Surv Ophthalmol. 2003;48(6):663-76.

3. Billson FH, Reich J, Hopkins IJ. Visual failure in a patient with ulcerative colitis treated by clioquinol. Lancet. 1972;1(7758):1015-6.

4. Greenstein AJ, Janowitz HD, Sachar DB. The extra-intestinal complications of Crohn's disease and ulcerative colitis: a study of 700 patients. Medicine (Baltimore). 1976;55(5):401-12.

5. Hopkins DJ, Horan E, Burton IL, Clamp SE, de Dombal FT, Goligher JC. Ocular disorders in a series of 332 patients with Crohn's disease. Br J Ophthalmol. 1974;58(8):732-7.

6. Knox DL, Schachat AP, Mustonen E. Primary, secondary and coincidental ocular complications of Crohn's disease. Ophthalmology. 1984;91(2):163-73.

7. Rankin GB, Watts HD, Melnyk CS, Kelly ML Jr. National Cooperative Crohn's Disease Study: extraintestinal manifestations and perianal complications. Gastroenterology. 1979;77(4 Pt 2):914-20.

8. Wright R, Lumsden K, Luntz MH, Sevel D, Truelove SC. Abnormalities of the sacro-iliac joints and uveitis in ulcerative colitis. Q J Med. 1965;34:229-36.

9. Fong AC, Schatz H, McDonald HR, Burton TC, Maberley AL, Joffe L, et al. Central retinal vein occlusion in young adults (papillophlebitis). Retina. 1992;12(1):3-11.

10. Hayreh SS. Optic disc vasculitis. Br J Ophthalmol. 1972;56(9):682-70.

11. Keyser BJ, Hass AN. Retinal vascular disease in ulcerative colitis. Am J Ophthalmol. 1994;118(3):395-6.

12. Talbot RW, Heppell J, Dozois RR, Beart RW Jr. Vascular complications of inflammatory bowel disease. Mayo Clin Proc. 1986;61(2):140-5.

13. Duker JS, Brown GC, Brooks L. Retinal vasculitis in Crohn's disease. Am J Ophthalmol. 1987;103(5):664-8.

14. Ruby AJ, Jampol LM. Crohn's disease and retinal disease. Am J Ophthalmol. 1990;110(4):349-53.

15. Lam A, Horda IT, Inwood MJ, Thompson S. Coagulation studies in ulcerative colitis and Crohn's disease. Gastroenterology. 1975;68(2):245-51.

16. Mayeux R, Fahn S. Strokes and ulcerative colitis. Neurology. 1978;28(6):571-4.

17. Kehoe EL, Newcomer KL. Tromboembolic phenomena in ulcerative colitis. Arch Intern Med. 1964;113:711-5. 